\title{
Patient Choice in Acute Care
}

\begin{abstract}
Consumer healthcare information plays a critical role in informing patients who participate in or make healthcare decisions for themselves without direct supervision of a healthcare professional. One such example is the choice of facility for acute care, prototypically between a fully equipped emergency care department (ED) at a hospital and a more convenient but less capable urgent care (UC) or retail clinic. We model a strategic patient making this decision taking into account the limited medical information and convenience factors that affect the patient's decision. This model is then used to inform the pricing decision made by the manager of the $\mathrm{UC}$. We show that a separating equilibrium, in which all patients self-triaged as noncritical choose to go to the UC first, dominates pooling equilibria for moderate error rates in self-triage. We analyze the separating equilibrium to examine the effect of consumer health information (CHI) systems, and show that as the quality of the CHI decreases and the error rates go up, the co-pay for an UC decreases, the facility is smaller, and makes less profit.
\end{abstract}

Keywords-consumer health information systems, classification error, Nash equilibrium, emergency room, urgent care

\section{INTRODUCTION}

Since the early 1980s in the US, some patients needing acute care, i.e., those needing short-term treatment for an injury, an episode of illness, or an urgent medical condition, have had a choice between a full fledged Emergency Department in a hospital and an Urgent Care facility that "delivers ambulatory medical care outside of a hospital emergency department on a walk-in basis, without a scheduled appointment ${ }^{1} . "$ The ED remains the most comprehensive acute care treatment facility, dealing with cases that require immediate care to non-urgent cases. UC centers typically only provide non-emergent care. In Table I we show the difference in the levels of care provided by EDs and UCs.

Urgent Care Centers have grown tremendously in popularity for providing acute care for non-emergent cases, i.e., for cases in levels 4 and 5 of Emergency Severity Index. The number of urgent care centers has grown from approximately 8,000 in 2008 to 9,300 in 2014 , with 4 or more urgent care centers in cities with population of 100,000 or more ${ }^{2}$.

Part of the reason for the rise of UC has been the congestion and wait times at emergency departments. Even as the patient demand for acute care has been increasing, the number of EDs has been decreasing in the U.S. (4). The net effect is that the wait times have been increasing explosively. US Centers for Disease Control (CDC) reports that between 2003 and 2009, the mean wait time to see a provider increased by $25 \%$ to

\footnotetext{
${ }^{1}$ This definition and the statistics on Urgent Care centers in this and the next paragraph come from the industry trade association - Urgent Care Association of America (UCAOA). See especially their results from a survey in 2013 (1) ${ }^{2}$ Ibid.
}

\begin{tabular}{|l|l|l|l|}
\hline $\begin{array}{l}\text { Emergency } \\
\text { Severity } \\
\text { Index } \\
(2)\end{array}$ & $\begin{array}{l}\text { Example of Condi- } \\
\text { tion }\end{array}$ & Percent & Providers \\
\hline $\begin{array}{l}\text { Level 1 - Im- } \\
\text { mediate }\end{array}$ & $\begin{array}{l}\text { resuscitation, cardiac } \\
\text { arrest, critically in- } \\
\text { jured trauma patient }\end{array}$ & $1.2 \%$ & ED only \\
\hline $\begin{array}{l}\text { Level 2 - } \\
\text { Emergent }\end{array}$ & $\begin{array}{l}\text { suicidal or homicidal } \\
\text { patient, chemotherapy } \\
\text { patient with fever, ac- } \\
\text { tive chest pain }\end{array}$ & $10.7 \%$ & ED only \\
\hline $\begin{array}{l}\text { Level 3 - Ur- } \\
\text { gent }\end{array}$ & $\begin{array}{l}\text { fractured ankle, } \\
\text { abdominal pain, } \\
\text { migraine }\end{array}$ & $42.3 \%$ & $\begin{array}{l}\text { ED and a } \\
\text { few UC }\end{array}$ \\
\hline $\begin{array}{l}\text { Level 4 - } \\
\text { Semi-urgent }\end{array}$ & $\begin{array}{l}\text { sore throat and fever, } \\
\text { stubbed toe, sprained } \\
\text { ankle, minor lacera- } \\
\text { tion }\end{array}$ & $35.5 \%$ & ED and UC \\
\hline $\begin{array}{l}\text { Level 5 - } \\
\text { Nonurgent }\end{array}$ & poison ivy, toothache & $8.0 \%$ & ED and UC \\
\hline
\end{tabular}

TABLE I: Levels of Care

almost an hour (5). The wait times are even worse for patients with non-emergent conditions. These patients, who make up a significant proportion of patients at an ED, $43 \%$ in the US in 2011 (3), are triaged into lower priority queues that have even longer wait times. These patients can be treated elsewhere such as in an UC (6) where the wait times are much lower. The wait time distribution at EDs and UCs are shown in Tables II and III. We note that only $3 \%$ of patients at an UC waited more than 40 minutes whereas $21.6 \%$ of the patients and ED waited an hour or more. Average wait times at each ED in US is available publicly, for instance at ER Wait Watcher ${ }^{3}$ which provides the average delay and travel time to nearby EDs from any address in US. A screen-shot is shown in Figure 1. Shifting some non-emergent patients from the ED to the UC can help all patients, but especially those who have nonemergent conditions.

While most Emergency Departments are affiliated with hospitals and are typically located in urban environments, Urgent Care centers are located in suburbs and in locations convenient for patients. The most common location for an UC is a shopping strip or a mall ${ }^{4}$.

The co-payments and fees charged by UCs are typically

\footnotetext{
${ }^{3}$ Published by Pro Publica at projects.propublica.org/emergency/ It uses annual wait time data gathered by Medicare

${ }^{4}$ According to a recent survey by the Urgent Care Association of America (UCAOA), the largest proportion, 38\% of Urgent care centers are located in a shopping center or strip mall.
} 


\begin{tabular}{|c|c|}
\hline Time to see a professional & \% of visits \\
\hline Fewer than 15 minutes & $27.0 \%$ \\
\hline 15-59 minutes & $40.7 \%$ \\
\hline 1 to 2 hours & $13.5 \%$ \\
\hline 2 to 3 hours & $4.8 \%$ \\
\hline 3 to 4 hours & $1.6 \%$ \\
\hline 4 to 6 hours & $1.0 \%$ \\
\hline 6 hours or more & $0.7 \%$ \\
\hline
\end{tabular}

TABLE II: Wait times at emergency departments

Source: US Center for Disease Control (3). Values do not ad up to $100 \%$ because of blank $(7.5 \%)$ and not applicable $(3.1 \%)$ data, and rounding.

\begin{tabular}{|c|c|}
\hline Time to see a professional & \% of visits \\
\hline Fewer than 20 minutes & $69 \%$ \\
\hline $21-40$ minutes & $28 \%$ \\
\hline 40 minutes or more & $3 \%$ \\
\hline
\end{tabular}

TABLE III: Wait time at urgent care centers

Source: Urgent Care Association of America Foundation (7) http://c.ymcdn.com/sites/ www.ucaoa.org/resource/resmgr/Files/UrgentCareMediaKit_2013.pdf

much lower than that charged by an ED. Consumer Reports in their April 2009 Health Report (8) reported that the copays and fees for UCs are comparable to that for a doctor visit, about $\$ 120$ on average, and much lower than at an ED, $\$ 400$ on average.

While UCs are more convenient and cheaper overall for nonemergent care, EDs still get non-emergent patients for a variety of reasons. Ability to pay is one reason. UCs only accept patients who have insurance or are willing to pay themselves. They do not accept medically indigent patients. These patients go to an ED which is required by law to accept them ${ }^{5}$. Another reason is availability. UCs are typically open during business hours from 8AM to 10PM, whereas the ED is open 24 hours a day. Yet another reason is mis-classification by the patient who thinks that she has an emergent condition but actually does not. We consider the quality of self-classification in greater detail below.

Patients requiring immediate treatment for extremely emergent conditions are often transported directly by ambulance services to the ED. From Table I we note that this makes up only about $1 \%$ of the patients at a typical ED. Other patients who have insurance coverage or are willing to pay for urgent care have a choice.

So how does a patient choose an acute care facility? The health insurance companies have a strong financial interest in making their insured patients aware of the UC option because it is much cheaper for them. Using the 2008 Medical Expenditures Panel Survey (MEPS), the Government Accountability Office reports that the average charge for a non-emergency

\footnotetext{
${ }^{5}$ In 1986 the US enacted a law 'Emergency Medical Treatment and Active Labor Act (EMTALA)' that requires hospitals accepting Medicare payments to provide emergency care treatment irrespective of ability to pay or legal status.
}

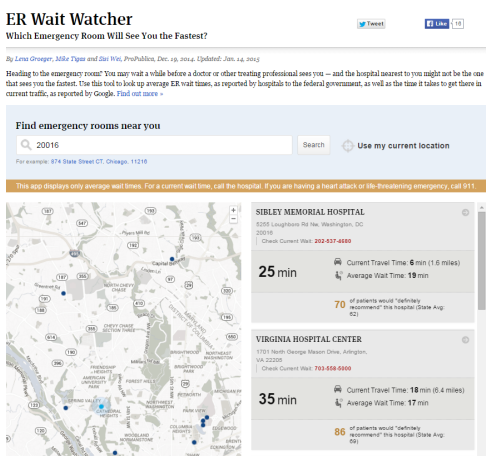

Fig. 1: Wait and travel times to near by Emergency

Departments provided by ER Wait Watcher http://projects.propublica.org/emergency/

visit to an ED is $\$ 2,101$ versus $\$ 203$ at a health care center (9). Many of the health insurance companies maintain websites and use other channels including mailings to inform the insureds of the UC option $(10,11)$. For instance, the health care insurance company Aetna Inc. reports that the average co-payment and out of pocket costs for sprains, influenza, minor lacerations and headaches (migraine and tension) is $\$ 550$ to $\$ 750$ in ED and $\$ 110$ to $\$ 150$ in UC (10) and that their patients can save money by choosing to go to an UC. Additionally, many potential patients go by Urgent Care centers in their usual day to day activities since many UC are located in malls, and other popular places.

The hard part in picking between an ED and an UC is deciding whether the patient's condition is emergent or not since UCs do not offer care for patients with emergent conditions (Emergency Severity Index 1 to 3 ). This is because the judgment is made by the patient or her family who typically are not medically trained. Again, most insurance companies and hospitals provide guidelines as a part of their consumer health information (CHI) initiative. An example is provided in Figure 2. Most insurance companies have similar CHI.

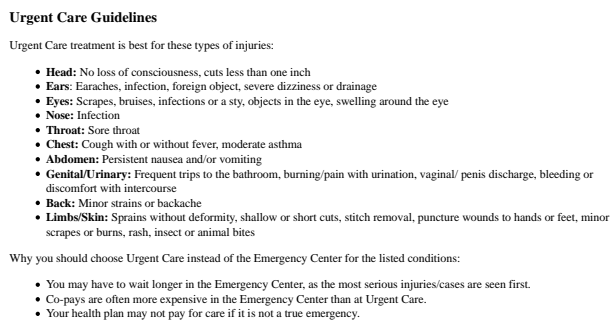

Fig. 2: A simple example for $\mathrm{CHI}$

Source: Mercy Health Cadillac http://www.mercyhealthcadillac.com/ urgent-care-guidelines

Self-triage into emergent and non-emergent conditions by the patient or their family is fraught with costly errors. Patients who go to an ED with non-emergent conditions will have longer waits and much larger co-pays. Patients who go to an UC with emergent conditions also have serious consequences that include delay of care for a serious life threatening 
condition, extra co-pays, and delay from additional travel. In this paper we model the self-triage activity as a process of classification with errors of both kinds. We consider a strategic patient who weighs all the costs, including waiting, co-pays, and the that of error in self-triage, in deciding whether to visit an UC or an ED.

In this paper we focus on the Urgent Care center and its management. In section (III) we model the behavior of a patient who is choosing an acute care facility on the basis of self-triage, expected waiting time and co-payment differences between ED and UC. We model the UC as a price taker, taking the price of the ED as a given and setting a discount for patients over the ED co-payment. We consider two kinds of patient behavior: separating equilibrium, the strategic patients who self triage as non-critical go to the UC and those that selftriage as critical go to the ED; while in the pooling equilibrium all the strategic patients go to the UC first ${ }^{6}$. We show that the separating equilibrium dominates the pooling equilibrium for moderate error rates.

In section (IV) we analyze the separating equilibrium. We characterize the discount set by the UC. In this equilibrium, the optimal discount set by the UC is increasing in both kinds of error, error in which a non-critical patient mistakenly thinks that she is critical and goes to the ED directly, and the error in which a truly critical patient mistakenly self-triages as noncritical and goes to the UC. Hence, the UC manager has an incentive to reduce errors.

\section{LiterATURE REVIEW}

The cost of waiting in healthcare settings is well document in literature. For instance, (12) studied the cost of increased wait times on society due to inappropriate use of the emergency department by patients seeking non-emergency or primary care. (13) mention that commonly studied effects of crowding included patient mortality, transport delays, treatment delays, ambulance diversion, patient elopement, and financial effect.

Models to predict waiting times for patients with differing priorities, such as between elective and acute care, have been developed starting the 1970 s $(12,14,15,16)$. This situation is similar to our setting for the emergency department in which critical patients have priority over non-critical patients. They find that the proportion of low and high priority patients is an important determinant of hospital performance.

There is extensive literature on the appointment scheduling of patients for outpatient and inpatient services. See especially the review by (17) and more recent papers (18), (19), (20), (21), (22), (23), (24), (25), (26), (27) and (28). While these papers shed light on the interaction between appointment scheduling and wait times, they are not directly relevant to our study because we examine the acute care setting in which appointments are not generally made or taken.

\footnotetext{
${ }^{6}$ The other pooling equilibrium in which all the patients first go the ED is not attractive to the UC since typically a patient at an ED does not leave and go to an UC.
}

In an interesting variation, (29) study the capacity reservation for urgent patients in primary care when there are both urgent and routine (nonurgent) patients. They define urgent cases as those that need to be seen in the same day as their request. They note that for some cases the patient's symptoms would lead the physician to categorize him as urgent. In other cases, it is more the patient' own sense of urgency that drives the categorization. They allow an urgent patient who cannot be served on the day of request to be seen the following day as an overflow. This is not generally acceptable for patients with extremely urgent cases (ESI 1 to 3 , as shown in Table I), and so we do not see these techniques employed in emergency rooms.

Few papers model choices by patients. One example is the paper by (30) in which the choice of a patient includes the appointment times, e.g., she can choose between the same day appointment in which different part of the day might be another choice or an appointment at a later day for a primary care clinic rather than the choice between medical facilities. The patient's choice of facility to go to is exogenous. Our model focuses on the facility choice.

In our model, the patient is picking between facilities based on a number of factors. Such strategic behavior by patients has been documented in literature. (31) note that patients, armed with information from the web, are increasingly involved in making healthcare decisions. Further, there is empirical evidence that the patients strategically weigh the different factors such as quality of care, waiting times, co-payments, in deciding what kind of care to get $(32,33)$. To these factors we add the cost of errors from self-classification, which itself is fraught with costly errors.

\section{THE MODEL}

Patients may or may not have a choice of which acute care facility to visit, an Emergency Department (ED) at a hospital or an Urgent Care center (UC), to visit. We label the patients who have the choice as strategic and those who do not as non-strategic. Though we include the non-strategic patients in the workflow to estimate wait times in each facility, we focus on strategic patients in the rest of this paper.

In this paper we divide the strategic patients into two groups: One labeled critical, is a group of patients with conditions with ESI (see Table I) in Levels 1, 2 and 3; and another group labeled non-critical is a group of patients with conditions with ESI 4 and 5. Let $\lambda_{0 c}$ and $\lambda_{0 n}$ be the arrival rates of non-strategic patients who are truly critical and non-critical, respectively. Similarly let $\lambda_{c}$ and $\lambda_{n}$ be the arrival rates of strategic patients who are truly critical and non-critical, respectively. A strategic patient's choice starts with self-triage or classification based on perceived symptoms and her prior medical knowledge including that obtained from consumer health information (CHI) systems.

The self-triage or classification done by a non-medical professional is fraught with errors. On the one hand, a patient who is truly critical, such as a heart attack patient thinking that the angina is upset stomach, may self-classify as non-critical. 
On the other hand, a patient who is truly non-critical, such as a patient with an upset stomach thinks that she has appendicitis, may self classify as critical. In either case, the patient is not fully informed or trained and may make an error. As we see from the examples above, two types of errors are possible: (1) A truly non-critical patient predicts that she is critical (Type I error) or (2) a truly critical patient predicts that she is noncritical (Type II error) ${ }^{7}$. We define $\alpha$ and $\beta$ as the error rates for Type I and Type II errors, respectively. Without loss of generality we take $\alpha+\beta \leq 1$ for if this is not the case then the labels in the guidelines can be switched to make it so. The confusion matrix, in Table IV exhibits the disposition of the patients between true and predicted classes.

\begin{tabular}{|c|c|c|}
\hline & Truly Critical & Truly Non-critical \\
\hline Predicts Critical & $(1-\beta) \lambda_{c}$ & $\alpha \lambda_{n}$ \\
\hline Predicts Non-critical & $\beta \lambda_{c}$ & $(1-\alpha) \lambda_{n}$ \\
\hline
\end{tabular}

TABLE IV: Confusion matrix

Patients incur a variety of costs in using the medical facility. Some costs, such as co-pays, are pecuniary while others, such as those associated with waiting and transfer, and effects on outcome because of delays, are non-pecuniary but important to consider. At the time of arrival patients incur co-pay $P$ in ED and $P-D$ in UC where $D$ is the discount offered by UC to patients. Patients who choose to go to UC but are truly critical incur the rerouting cost $R$ from UC to ED. These patients incur both co-pays.

Patients who arrive at a facility may wait before they are treated, and treatment itself may take a while. For separating equilibrium, we define the expected system waiting times as $W_{c}$ and $W_{n}$ for critical and non-critical patients in ED, respectively and $W$ for non-critical patients in $\mathrm{UC}^{8}$. We also assign patient waiting cost rate $c_{c}$ and $c_{n}$ for critical and non-critical patients, respectively. Putting these together we determine the expected disutility that are shown in the Table $\mathrm{V}$ below.

\begin{tabular}{|c|c|c|}
\hline & Critical & Non-critical \\
\hline Go to ED & $P+c_{c} W_{c}$ & $P+c_{n} W_{n}$ \\
\hline Go to UC & $2 P-D+R+c_{c} W_{c}$ & $P-D+c_{n} W$ \\
\hline
\end{tabular}

TABLE V: Expected Cost incurred by a Strategic Patient for Separating Equilibrium

The UC manager influences the choice of the patients by setting the discount. Two possible outcomes are analyzed:

\footnotetext{
${ }^{7}$ In statistics Type I and Type II errors refer to the false positive and the false negative errors, respectively. We treat the critical class as the positive class for purposes of assigning Type I and Type II error names.

${ }^{8}$ For ease of exposition, here we consider the separating equilibrium. For pooling equilibrium, the expected system times are $W_{c}^{\prime}$ and $W_{n}^{\prime}$ for critical and non-critical patients in ED, respectively and $W^{\prime}$ for non-critical patients in UC.
}

1) A Separating Equilibrium in which all the patients who self-triage as non-critical go to the UC, and all the patients who self-triage as critical go to the $\mathrm{ED}^{9}$.

2) A Pooling Equilibrium in which all the strategic patients go first to the UC, and then the critical ones are triaged to the $\mathrm{ED}^{10}$.

These equilibria are examined in sections (III-A) and (III-B).

\section{A. The Separating Equilibrium}

In this section we explore a separating equilibrium in which strategic patients who self-triage as non-critical choose to go to UC instead of the ED. However, because of error in self triage, only a $(1-\alpha)$ fraction of non-critical patients go to the UC. The remainder, $\alpha \lambda_{n}$, go to the ED thinking they are critical. Similarly, $(1-\beta) \lambda_{c}$ of the critical patients go to the ED directly, and $\beta \lambda_{c}$ erroneously thinking that they are noncritical go first to the UC and then are re-routed to the ED. The care map is shown in the top part of Figure 3.

Including the non-strategic patient flows of $\lambda_{0 c}$ and $\lambda_{0 n}$ for critical and non-critical condition, the total net flows into the different facilities are:

$$
\begin{aligned}
& \text { Critical patients into ED: } \lambda_{c}^{E}=\lambda_{0 c}+\lambda_{c} \\
& \text { Non-critical patients into ED: } \lambda_{n}^{E}=\lambda_{0 n}+\alpha \lambda_{n} \\
& \text { Non-critical patients into UC: } \lambda^{U}=(1-\alpha) \lambda_{n} .
\end{aligned}
$$

We also define service rates in both acute care facilities. We define $\mu$ as the service rate for non-critical patients in UC, and $\theta$ and $\nu$ as the service rate for critical and non-critical patients in ED, respectively. Further, we assume that $\nu>\theta$, i.e., it takes longer to provide healthcare service to a critical patient than a non-critical patient.

A separating equilibrium, in which strategic patients who are predicted to be non-critical use the UC, is characterized next. Let $C$ and $N C$ be events where patients are truly critical and non critical, respectively and $C^{\prime}$ and $N C^{\prime}$ be the events that they are predicted critical and predicted noncritical, respectively. Let $\pi$ denote the precision of the noncritical classification, i.e, $\pi=\operatorname{Pr}\left[N C \mid N C^{\prime}\right]$ and $\pi^{\prime}$ denote the precision of the critical classification, i.e., $\pi^{\prime}=\operatorname{Pr}\left[C \mid C^{\prime}\right]$.

Examining the confusion matrix in Table IV, we get the precisions for the non-critical and critical classes, respectively, as follows:

$$
\pi=\frac{(1-\alpha)(1-\chi)}{\beta \chi+(1-\alpha)(1-\chi)} \text { and } \pi^{\prime}=\frac{(1-\beta) \chi}{(1-\beta) \chi+\alpha(1-\chi)}
$$

where $\chi=\frac{\lambda_{c}}{\lambda_{c}+\lambda_{n}}$ is the prior probability of critical condition for strategic patients.

\footnotetext{
${ }^{9}$ The other separating equilibrium, in which the patients self-triaged as critical go to the UC, and those self-triaged non-critical go to the ED is dominated for the patients by the separating equilibrium examined here.

${ }^{10}$ The pooling equilibrium in which all the strategic patients go the ED is dominated for the UC manager by the pooling equilibrium examined above. Since very few patients ever leave an ED to go to an UC, in the latter case, the UC would get almost no patients.
} 
Separating Equilibrium (predicted critical go to ED, predicted non-critical go to UC

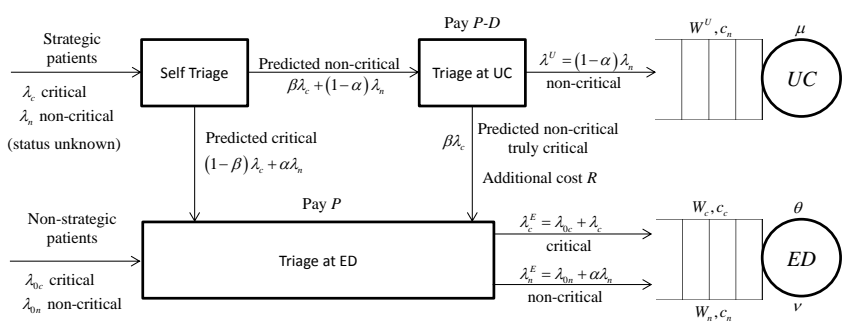

Pooling Equilibrium (all strategic patients go to UC)

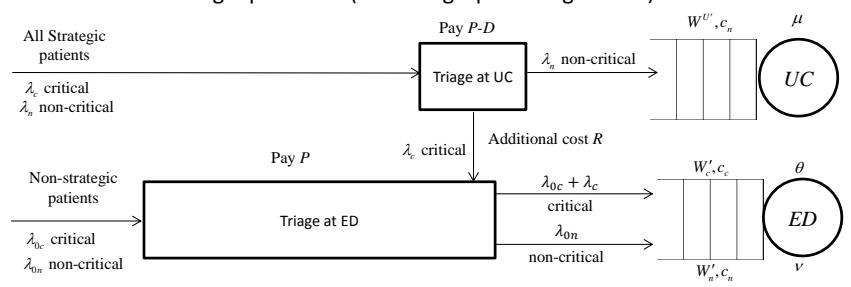

Notation:

$\begin{array}{ll}\lambda_{c} & \text { Arrival rate of strategic patients who are }\end{array}$ truly critical

$\lambda_{n} \quad$ Arrival rate of strategic patients who are truly non-critical

$\lambda_{0 c} \quad$ Arrival rate of non-strategic patients who are truly critical

$\lambda_{0 n} \quad$ Arrival rate of non-strategic patients who are truly non-critical

$\alpha \quad$ The error rate of patients who are classified critical but are truly non-critical

$\beta \quad$ The error rate of patients who are classified non-critical but are truly critical

$P \quad$ Co-payment for ED services

$D \quad$ Discount in co-payment for UC services

$P-D \quad$ Co-payment net of discount for UC services

$\mu \quad$ The service rate at UC

$\theta \quad$ The service rate for critical patients at ED

$\nu \quad$ The service rate for non-critical patients at ED

$W \quad$ The expected time in system for patients at UC in separating equilibrium

$W_{c} \quad$ The expected time in system for critical patients at ED in separating equilibrium

$W_{n} \quad$ The expected time in system for noncritical patients at ED in separating equilibrium

$W^{\prime} \quad$ The expected time in system for patients at $\mathrm{UC}$ in pooling equilibrium

$W_{c}^{\prime} \quad$ The expected time in system for critical patients at ED in pooling equilibrium

$W_{n}^{\prime} \quad$ The expected time in system for noncritical patients at ED in pooling equilibrium

$c_{c} \quad$ Waiting cost rate for critical patients

$c_{n} \quad$ Waiting cost rate for non-critical patients
Theorem 1. A separating equilibrium, in which predicted noncritical patients go to UC and predicted critical patients go to $E D$, is obtained if and only if

$$
\begin{aligned}
&(1-\pi)(P+R)-\pi c_{n}\left(W_{n}-W\right) \leq D \\
& \leq \pi^{\prime}(P+R)-\left(1-\pi^{\prime}\right) c_{n}\left(W_{n}-W\right) .
\end{aligned}
$$

Further if $\alpha+\beta \leq 1$ and $W_{n}-W \geq 0$, then there exists a $D$ that satisfies both inequalities in (1).

The first inequality in Theorem 1 reflects the requirement that patients self-triaged as non-critical go to the UC. The second inequality reflects the requirement that the patients selftriaged as critical go to the ED. The proofs of all theorems and propositions in this paper are given in the Appendix.

Any discount $D$ that satisfies the inequalities in Theorem 1 will result in a separating equilibrium. However, the UC administration prefers lower discounts. Hence, the lower bound is binding for the un-dominated separating equilibrium.

Corollary 2. The lowest discount, $D^{*}$, offered by a profit maximizing $U C$ is

$$
D^{*}=(1-\pi)(P+R)-\pi c_{n}\left(W_{n}-W\right)
$$

Intuitively, the corollary reflects the fact that only the first inequality in Theorem 1 is binding. This sets the discount $D$ to be such that the patient triaged as non-critical is indifferent between going to the UC and ED. The first term is the expected penalty from rerouting the patients who are predicted noncritical but are truly critical. The second term is the benefit arising from a reduction in expected waiting cost of going to an UC over an ED.

For tractability and ease of exposition, we assume that the expected system times at the ED arise from an M/M/1 queuing discipline with non-preemptive priority for critical patients. Following (34) and using the arrival and service rates defined above, the expected system waiting times are exhibited below.

$$
\begin{aligned}
& W_{c}=\frac{\rho_{c} / \theta+\rho_{n} / \nu}{1-\rho_{c}}+\frac{1}{\theta} \\
& W_{n}=\frac{\rho_{c} / \theta+\rho_{n} / \nu}{\left(1-\rho_{c}\right)\left(1-\rho_{c}-\rho_{n}\right)}+\frac{1}{\nu}
\end{aligned}
$$

where $\rho_{n}=\lambda_{n}^{E} / \nu$, and $\rho_{c}=\lambda_{c}^{E} / \theta$. Similarly, we assume an M/M/1 queuing discipline for the UC. Then the expected system waiting time in UC is exhibited below.

$$
W=\frac{1}{\mu-(1-\alpha) \lambda_{n}}
$$

We also assume that the ED is more congested than UC, i.e., $W_{n} \geq W$.

\section{B. Pooling Equilibrium}

In this equilibrium, all the strategic patients go to the UC first. They pay the co-pay and are triaged. Patients who are triaged critical then go to the ED for treatment, incurring the additional co-pay at the ED and the cost of re-routing. The

Fig. 3: Preliminaries of the Model 
care-map is shown in the bottom part of Figure 3. The flow of patients to be treated in this equilibrium are:

Net flow rate of critical patients into ED: $\lambda_{0 c}+\lambda_{c}$

Net flow rate of non-critical patients into ED: $\lambda_{0 n}$

Net flow rate of non-critical patients into UC: $\lambda_{n}$.

With these flows, and again assuming that the expected system times at the ED arise from an $\mathrm{M} / \mathrm{M} / 1$ queuing discipline with non-preemptive priority for critical patients, we get the following expected system waiting times:

$$
\begin{aligned}
W_{c}^{\prime} & =\frac{\rho_{c} / \theta+\rho_{n}^{\prime} / \nu}{1-\rho_{c}}+\frac{1}{\theta} \\
W_{n}^{\prime} & =\frac{\rho_{c} / \theta+\rho_{n}^{\prime} / \nu}{\left(1-\rho_{c}\right)\left(1-\rho_{c}-\rho_{n}^{\prime}\right)}+\frac{1}{\nu}
\end{aligned}
$$

where $\rho_{n}^{\prime}=\lambda_{0 n} / \nu$.

Similarly, we assume an M/M/1 queuing discipline for the UC. Then the expected system waiting time in UC is exhibited below.

$$
W^{\prime}=\frac{1}{\mu-\lambda_{n}}
$$

We again assume that the ED is more congested than the UC, i.e., $W_{n}{ }^{\prime} \geq W^{\prime}$. The UC manager sets the discount to induce all strategic patients to go to the UC. This discount is determined in the theorem below.

Theorem 3. A pooling equilibrium, in which all strategic patients go to UC first, is obtained if and only if the discount $D^{\prime}$ is such that:

$$
\pi^{\prime}(P+R)-\left(1-\pi^{\prime}\right) c_{n}\left(W_{n}^{\prime}-W^{\prime}\right) \leq D^{\prime}
$$

A number of points are worth noting:

1) We show that the self-triage is informative. Since it is also costless, the patients will perform the free self-triage before deciding on course of action.

2) The condition that patients self-triaged as non-critical preferring the UC over the ED is dominated by the condition that patients self-triaged as critical also prefer the UC over the ED.

3) The UC manager will pick the smallest discount possible as that increases the profit. Hence, the discount, $D^{\prime}$ will be picked such that condition (4) is binding.

\section{Comparing the Separating and Pooling Equilibria}

The discount needed to get all strategic patients to go to the UC is higher than that needed to get only those predicted to be non-critical to go to the UC. Hence the discount needed to obtain the pooling equilibrium is greater than that needed to obtain the separating equilibrium. This is shown in the proposition below.

Proposition 4. The discount needed to obtain the pooling equilibrium in which all strategic patients prefer to go to the $U C, D^{\prime}$ is greater than the discount needed to obtain the separating equilibrium in which the patients predicted noncritical prefer to go to the $U C, D^{*}$.

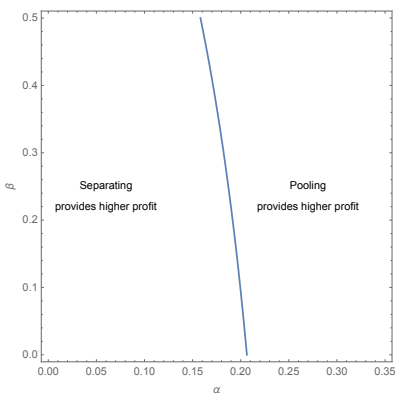

Parameters values: $\theta=50, \nu=100, \mu=100, \lambda_{0 c}=2, \lambda_{0 n}=20, \lambda_{c}=12, \lambda_{n}=50$, $I=200, P=100, R=100, c_{n}=50$

Fig. 4: Profits from the Equilibria

An UC center typically receives a payment from a thirdparty, such as a health insurance company, for each patient treated at the center. Let $I$ be the payment per patient. This is independent of the error rates $\alpha$ and $\beta$. With this payment, the short-run profit for the UC center in each of the equilibrium is shown below.

Profit in the separating equilibrium = $\left((1-\alpha) \lambda_{n}+\beta \lambda_{c}\right)\left(P-D^{*}+I\right)$

Profit in the pooling equilibrium $=\left(\lambda_{n}+\lambda_{c}\right)\left(P-D^{\prime}+I\right)$

We note that the flow of patients is greater in the pooling equilibrium but the revenue per patient is lower because of the higher discount. The net effect is shown in the Figure 4 below.

In Figure 4 we note that for moderate error rates, the separating equilibrium provides greater profit for the UC. In the following sections we assume that the error rates are such that the separating equilibrium is the one of interest.

\section{Analysis of the Discount in the SeParating EQUILIBRIUM}

In this section we analyze the effect of error rates on the discount offered by the UC manager in the separating equilibrium in which all the patients self-triaged as non-critical go to the UC. The two error rates are: Type I Error Rate $(\alpha)$ which is the rate at which non-critical patients are classified as critical; and the Type II Error Rate $(\beta)$ which is the rate at which the critical patients are classified as non-critical. We re-state equation (2) highlighting the terms that are a function of $\alpha$ and $\beta$.

$$
D^{*}(\alpha, \beta)=(1-\pi(\alpha, \beta))(P+R)-\pi(\alpha, \beta) c_{n} \Delta W(\alpha)
$$

where $\Delta W=W_{n}-W$ and $\pi=\operatorname{Pr}\left[N C \mid N C^{\prime}\right]$ is the precision of the non-critical classification.

The precision of non-critical classification $\pi(\alpha, \beta)=$ $\frac{(1-\alpha)(1-\chi)}{\beta \chi+(1-\alpha)(1-\chi)}$ is decreasing in both $\alpha$ and $\beta$. This is shown in Figure 5 below. Since $\pi$ enters in with a negative sign in Equation (5), the discount is increasing in both the error rates.

Now we turn to the effect of error rates on the $\Delta W$ term. It is only affected by $\alpha$, the rate at which a non-critical patient 


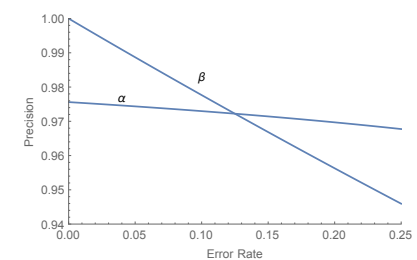

Parameters values: Type I $\alpha$ varying, $\beta=0.125$; Type II $\beta$ varying, $\alpha=0.125, \chi=0.5$.

Fig. 5: Precision, $\operatorname{Pr}\left[N C \mid N C^{\prime}\right]$, vs. Type I and II Error Rates

mistakenly classifies herself as critical ${ }^{11}$. Increasing $\alpha$ results in more non-critical patients ending up at the ED rather than UC, effectively shifting traffic from the UC to the ED. In more detail, the flow of strategic non-critical patients is split between the ED at rate $\alpha \lambda_{n}$ and $\mathrm{UC}$ at the rate $(1-\alpha) \lambda_{n}$. As the Type I error rate $\alpha$ increases, the ED gets more congested, UC gets less congested and the waiting time difference between the two, $\Delta W$ increases. This is shown in Figure 6.

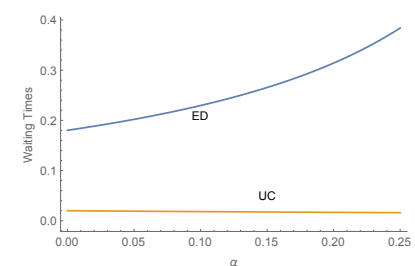

Parameters values: $\theta=30, \nu=120, \mu=100, \lambda_{0 c}=4, \lambda_{0 n}=40, \lambda_{c}=10, \lambda_{n}=50$

Fig. 6: Expected System Times in ED and UC vs. Type I Error Rate

Summing up the discussion above, $\beta$ only affects the precision and an increase in $\beta$ results in a bigger discount. In contrast, the net effect of an increase in $\alpha$ will depend on the competing effects on the precision $\pi$, and the waiting time difference $\Delta W$. When the ED is not too congested compared to the UC, the slope of the difference in waiting time with respect to traffic and $\alpha$ is not too large and the effect on $\pi$ dominates. In this case the discount will be increasing in $\alpha$. However, if the ED is much more congested than the UC, a transfer of a non-critical patient from the UC to the ED because of Type I error will cause a much larger increase in waiting time at ED and not much of decrease in waiting time in the UC, i.e., the effect of $\alpha$ on $\Delta W$ will swamp out the effect on $\pi$ and the discount may decrease with increasing $\alpha$. These results are formally presented in Proposition 5 below.

Proposition 5. The discount is increasing in $R$ and $\beta$. If the $E D$ is not too congested so that

$$
\frac{\rho_{n}}{\left(1-\rho_{n}-\rho_{c}\right)}\left(W_{n}+W_{c}\right) \leq \frac{\beta \chi(P+R)}{c_{n}(1-\alpha)^{2}}-\lambda_{n} W^{2}
$$

then the discount is also increasing in $\alpha$.

\footnotetext{
${ }^{11}$ The other error rate $\beta$, in which a critical patient classifies herself as critical affects the discount only through the expected penalty of rerouting and delays are not affected by it as after re-routing all critical patients end up at the ED.
}

Proposition 5 is illustrated by the graphs below. We will vary $\lambda_{0 c}$, the flow of non-strategic critical patients to vary the amount of congestion at the ED. This is a good parameter to vary the overall congestion of the ED as it affects all the delay times without directly affecting other statistics. In Figure 7 we show the critical level of $\lambda_{0 c}$ for satisfying equation (6).

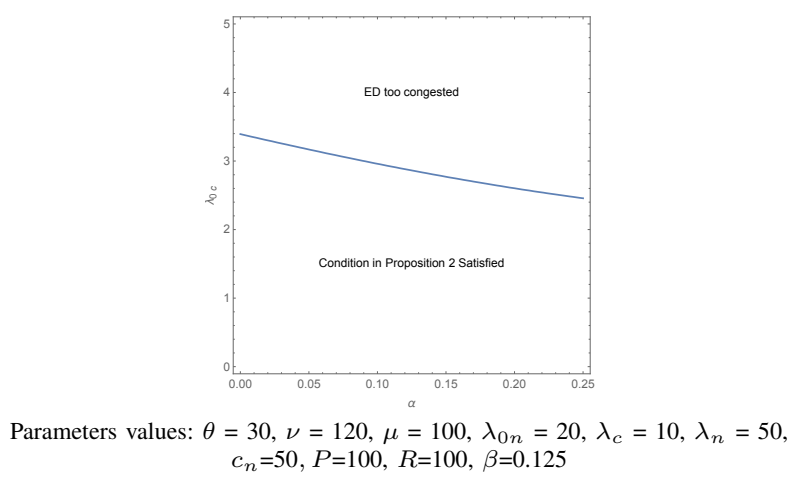

Fig. 7: Critical Level of Non-Strategic Critical Patients Traffic to Satisfy Proposition 5

The graphs of the optimal discount Vs. $\alpha$ are shown for $\lambda_{0 c}=1,2,4$ are shown in Figure 8. The condition (6) is slack for values 1 and 2 and these instances we see that the discount is increasing in $\alpha$. For $\lambda_{0 c}=4$, a value at which the $\mathrm{ED}$ is too congested and condition 6 is violated, the discount is decreasing in $\alpha$.

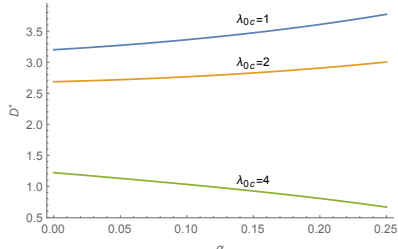

Parameters values: $\theta=30, \nu=120, \mu=100, \lambda_{0 n}=20, \lambda_{c}=10, \lambda_{n}=50$, $c_{n}=50, P=100, R=100, \beta=0.125$

Fig. 8: Discount vs. Type I Error Rate and ED Congestion

The effect of Type II error rate $\beta$ is shown in Figure 9. The main effect of this error on the discount is via the penalty term, which is increasing in $\beta$ and the rerouting cost $R$. So to achieve a lower discount UC administration can either focus on reducing the Type II error rate or the rerouting cost. Rerouting cost can be largely reduced by choosing the location of UC closer to an ED. Nevertheless, UCs can reduce rerouting costs in several ways. For instance, UC finishes the triage eliminating the need for another triage in ED, calls ED and assigns the required doctors to the patient and also transport the patient to ED. On the other hand, UC can reduce $\beta$ by increasing the quality of CHI.

Finally, we combine the two effects by illustrating the change in the regions in which separating equilibrium exists for different discount values as a function of error rates in Figure 10. As expected, as discount increases, separating equilibrium exists for a larger set of error rate values. This 


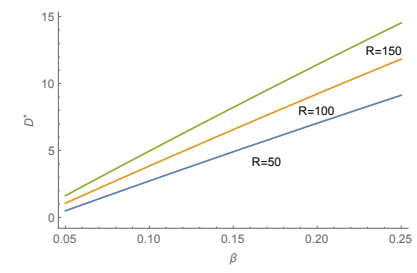

Parameters values: $\theta=30, \nu=120, \mu=100, \lambda_{0 c}=2, \lambda_{0 n}=40, \lambda_{c}=10, \lambda_{n}=50$, $c_{n}=15, P=150, \alpha=0.125$

Fig. 9: Discount vs. Type II Error Rate and Rerouting Cost

is because as the discount increases, patients are more incentivized to go to UC.

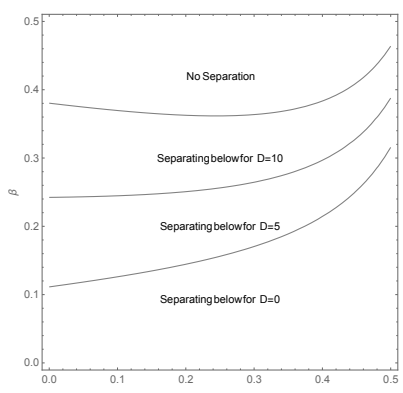

Parameters values: $\theta=30, \nu=120, \mu=100, \lambda_{0 c}=2, \lambda_{0 n}=40, \lambda_{c}=10, \lambda_{n}=50$ $c_{n}=50, P=150, R=50$

Fig. 10: Separating Equilibrium vs. Error Rates and Discount

\section{MANAGERIAL IMPLICATIONS AND CONCLUSION}

We built a model of patient's choice of acute care facility, an Emergency Department or an Urgent Care center, that includes not only the wait times and co-payment differences between the facilities, but also the impact of errors in self-classification and subsequent choice of the facility.

We showed that the errors in self-triage can have significant impacts on the choice of an acute care facility by the patient, the co-payment at an Urgent Care center, the capacity and profits generated by an UC in the long run, and the social welfare of all patients.

A reduction in Type I error rate, the error rate at which noncritical patients classify themselves as critical, appropriately increases the traffic at the UC by shifting more of the noncritical patients to the $\mathrm{UC}$, reduces the wait times at EDs which are typically more congested than UCs, while modestly increasing the waiting time at UCs. Overall, unless the ED is less congested than the UC so that a shift of traffic because of Type I error from the UC to the ED improves waiting times through load balancing - a rare situation indeed, UC can charge a higher co-pay, improve profits and capacity, and ultimately the social welfare for all patients also increases as this error rate decreases.

The effect of a reduction in Type II error rate, the error rate at which a critical patient is self-classified as non-critical, is more straight forward. A reduction in this error rate allows for a critical patient to get more immediate care using the full capabilities of the ED. This improvement is then factored in by the patient, and it allows the UC to charge a higher co-pay.

So generally, all the participants in the acute care system, the patients, the ED, the UC are better off with lower error rates. Since the decision is made by the patient in the field, two major hindrances remain to reduce errors: One is that the decision is made by the patient who is typically medically untrained and the other is the lack of instrumentation to provide vital health information. Both of these can be ameliorated with technology and better practices.

First, the guidelines could be improved. A systematic study of guidelines in different regions and the effect on error rates may help in improving them. Second, medically trained personnel could be involved earlier in the decision making process. Indeed, some healthcare insurers provide phone numbers with healthcare personnel standing by to help with this decision. While this helps, they are still hindered by lack of instrumentation which can provide important health data. As an example of this hindrance, we note that blood oxygen saturation is an important determinant for urgency of care and yet most patients do not have easy and immediate access to instruments that can measure this. But this situation is changing. Modern health fitness devices are increasingly able to perform non-invasive measurements in real time and transmit them to the cloud for further analysis. These could be used to dramatically reduce the error rates and improve social welfare.

\section{REFERENCES}

[1] UCAOA, "Benchmarking survey headlines summary," Urgent Care Association of America, 2014.

[2] AHRQ, Emergency Severity Index (ESI): A Triage Tool for Emergency Department: Implementation Handbook. Agency for Healthcare Research and Quality, 2014.

[3] CDC, National Hospital Ambulatory Medical Care Survey: 2011 Emergency Department Summary Tables. Centers for Disease Control/National Center for Health Statistics, 2011. [Online]. Available: http://www.cdc.gov/nchs/data/ahcd/ nhamcs_emergency/2011_ed_web_tables.pdf

[4] Avalere, "Trendwatch chartbook 2014: Trends affecting hospitals and health systems," 2012.

[5] E. Hing and F. Bhuiya, "Wait time for treatment in hospital emergency departments: 2009," NCHS Data Brief, No. 102, 2012.

[6] R. M. Weinick, R. M. Burns, and A. Mehrotra, "Many Emergency Department Visits Could Be Managed At Urgent Care Centers And Retail Clinics," Health Affairs, vol. 9, pp. 1630 1636, 2010.

[7] Anderson, Niebuhr and Associates, Inc, "2012 Urgent Care Benchmarking Study Results," Urgent Care Association of America, Tech. Rep., 2012. [Online]. Available: http://c.ymcdn.com/sites/www.ucaoa.org/resource/ resmgr/Files/UrgentCareMediaKit_2013.pdf

[8] CR, "Urgent care: What you need to know," Consumer Reports on Health, 2009. [Online]. Available: http://www.consumerreports.org/cro/2013/01/ urgent-care-what-you-need-to-know/index.htm

[9] GAO, "Hospital emergency departments: Health center strategies that may help reduce their use," GAO Report, vol. GAO11-643T, 2011. 
[10] Aetna Inc., "Say goodbye to unnecessary ER visits," Aetna Inc. Web Site, 2014. [Online]. Available: http://www.aetna.com/ docfind/cms/assets/pdf/Urgent_Care.pdf

[11] BlueCross BlueShield Association, "Alternatives to Emergency Room Care," BlueCross BlueShield Association Web Site, 2015. [Online]. Available: http://www.bcbs.com/why-bcbs/ immediate-medical-care/alternatives-to-emergency.html

[12] K. Siddharthan, W. J. Jones, and J. A. Johnson, "A priority queuing model to reduce waiting times in emergency care," International Journal of Health Care Quality Assurance, vol. 9, no. 5, pp. 10-16, 1996.

[13] N. R. Hoot and D. Aronsky, "Systematic review of emergency department crowding: causes, effects, and solutions," Annals of emergency medicine, vol. 52, no. 2, pp. 126-136, 2008.

[14] W. Shonick and J. R. Jackson, "An improved stochastic model for occupancy-related random variables in general acute hospitals." Operations Research, vol. 21, no. 4, pp. 952-965, 1973.

[15] B. Fries, "Bibliography of operations research in health-care systems." Operations Research, vol. 24, no. 5, p. 801, 1976.

[16] L. Green, "Queueing analysis in healthcare," in Patient flow: reducing delay in healthcare delivery. Springer, 2006, pp. 281-307.

[17] T. Cayirli and E. Veral, "Outpatient scheduling in health care: a review of literature," Production and Operations Management, vol. 12, no. 4, pp. 519-549, 2003.

[18] L. V. Green, S. Savin, and B. Wang, "Managing patient service in a diagnostic medical facility," Operations Research, vol. 54, no. 1, pp. 11-25, 2006.

[19] L. R. LaGanga and S. R. Lawrence, "Clinic overbooking to improve patient access and increase provider productivity*," Decision Sciences, vol. 38, no. 2, pp. 251-276, 2007.

[20] X. Qu, R. L. Rardin, J. A. S. Williams, and D. R. Willis, "Matching daily healthcare provider capacity to demand in advanced access scheduling systems," European Journal of Operational Research, vol. 183, no. 2, pp. 812-826, 2007.

[21] J. Patrick, M. L. Puterman, and M. Queyranne, "Dynamic multipriority patient scheduling for a diagnostic resource," $O p$ erations research, vol. 56, no. 6, pp. 1507-1525, 2008.

[22] L. W. Robinson and R. R. Chen, "A comparison of traditional and open-access policies for appointment scheduling," Manufacturing \& Service Operations Management, vol. 12, no. 2, pp. 330-346, 2010.

[23] N. Liu, S. Ziya, and V. G. Kulkarni, "Dynamic scheduling of outpatient appointments under patient no-shows and cancellations," Manufacturing \& Service Operations Management, vol. 12, no. 2, pp. 347-364, 2010.

[24] B. Zeng, A. Turkcan, J. Lin, and M. Lawley, "Clinic scheduling models with overbooking for patients with heterogeneous noshow probabilities," Annals of Operations Research, vol. 178, no. 1, pp. 121-144, 2010.

[25] A. Saure, J. Patrick, S. Tyldesley, and M. L. Puterman, "Dynamic multi-appointment patient scheduling for radiation therapy," European Journal of Operational Research, vol. 223, no. 2, pp. 573-584, 2012.

[26] L. R. LaGanga and S. R. Lawrence, "Appointment overbooking in health care clinics to improve patient service and clinic performance," Production and Operations Management, vol. 21, no. 5, pp. 874-888, 2012.

[27] J. Feldman, N. Liu, H. Topaloglu, and S. Ziya, "Appointment scheduling under patient preference and no-show behavior," Operations Research, vol. 62, no. 4, pp. 794-811, 2014.
[28] C. Zacharias and M. Pinedo, "Appointment scheduling with noshows and overbooking," Production and Operations Management, vol. 23, no. 5, pp. 788-801, 2014.

[29] G. Dobson, S. Hasija, and E. J. Pinker, "Reserving capacity for urgent patients in primary care," Production and Operations Management, vol. 20, no. 3, pp. 456-473, 2011.

[30] D. Gupta and L. Wang, "Revenue management for a primarycare clinic in the presence of patient choice," Operations Research, vol. 56, no. 3, pp. 576-592, 2008.

[31] L. Baker, T. Wagner, S. Singer, and M. Bundorf, "Use of the internet and e-mail for health care information: Results from a national survey," JAMA, vol. 289, no. 18, pp. 2400-2406, 2003.

[32] J. Nyman, "Excess demand, consumer rationality, and the quality of care in regulated nursing homes," Health Services Research, vol. 24, pp. 105-127, 1989.

[33] D. Padgett and B. Brodsky, "Psychosocial factors influencing non-urgent use of the emergency room: A review of the literature and recommendations for research and improved service delivery," Social Science \& Medicine, vol. 35, no. 9, pp. 1189 - 1197, 1992.

[34] L. Kleinrock, Queueing Systems. Wiley Interscience, 1976, vol. II: Computer Applications. 